\title{
Effects of analgesic strategies on postoperative acute or chronic pain, delirium and complications in laparoscopic colorectal surgery
}

\author{
Yan Jiang ${ }^{1}$, Xiao-Long Liang ${ }^{1}$, Shu-Fang Sun ${ }^{1}$, and Hong Liu ${ }^{1}$ \\ ${ }^{1}$ Affiliation not available
}

July 22, 2020

\begin{abstract}
Aims: Postoperative pain and complications are the main risk factors against patients' recovery after colorectal surgery, and the pain can aggravate postoperative complications. This study aimed to investigate the effects of various postoperative analgesic strategies on the recovery after laparoscopic colorectal surgery. Methods: 400 patients undergoing laparoscopic colorectal cancer surgery were recruited, 335 patients were included eventually and five groups were involved: PCEA with ropivacaine in group ropivacaine, and PCIA with morphine, nalbuphine, sufentanil, or flurbiprofen plus sufentanil in the other four groups. The postoperative delirium (POD), postoperative pain, stress, and complications were recorded. Results: The incidence of POD and chronic postsurgical pain (CPSP) at 3 months or 6 months after surgery were higher in group sufentanil $(\mathrm{P}=0.004, \mathrm{P}=0.003$ or $\mathrm{P}=0.003$, vs. group ropivacaine). The risks of movement-evoked pain (MEP) were much higher in group nalbuphine, group sufentanil and group flurbiprofen plus sufentanil $(\mathrm{P}=0.001, \mathrm{P} j 0.001$, and $\mathrm{P} j 0.001$, vs. group ropivacaine ), but not in group morphine. There were no differences in cortisol and ACTH levels in the 5 groups $\left(\mathrm{P}_{\dot{0} 0.05)}\right.$. The incidence of pruritus was found significantly higher in group morphine than in the other groups except group $\mathrm{S}(\mathrm{P} ; 0.005)$. CPSP and POD were associated with MEP ( $\mathrm{P} ; 0.05$ ). Conclusion: PCEA is the first choice for postoperative analgesia in laparoscopic colorectal surgery, PCIA with morphine or nalbuphine can be considered an alternative, but sufentanil or combined with flubiprofen might not be a good choice.
\end{abstract}

Effects of analgesic strategies on postoperative acute or chronic pain, delirium and complications in laparoscopic colorectal surgery

Running title: Analgesic strategies in colorectal surgery

Yan Jiang11Joint first author: Yan Jiang, Xiao-Long Liang, Shu-Fang Sun (They contributed equally to this work) Chongqing University Cancer Hospital, Chongqing, 400030, China, MM, Xiao-Long Liang ${ }^{1}$, MM, Shu-Fang Sun ${ }^{1}$, MM, Hong-Liang Liu22Corresponding author: Hong-Liang Liu Chongqing University Cancer Hospital, Chongqing, 400030, China Tel: +86 13883686721, Fax: 023-65075692, E-mail address:liuhl75@163.comAddress correspondence to Hong-Liang Liu, MD, PhD, No. 181 Hanyu Road, Shapingba District, Chongqing 400030, P.R. China, MD, PhD

Chongqing University Cancer Hospital, Chongqing, 400030, China

Acknowledgments: None.

\section{Hosted file}

manuscript.docx available at https://authorea.com/users/345108/articles/471435-effects-ofanalgesic-strategies-on-postoperative-acute-or-chronic-pain-delirium-and-complicationsin-laparoscopic-colorectal-surgery 


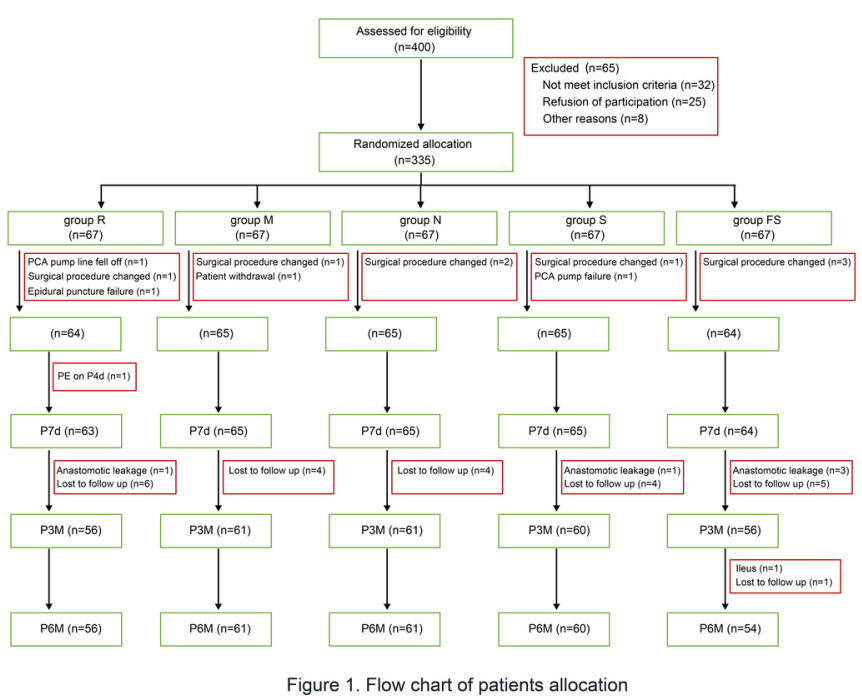

Note: pulmonary embolism (PE); postoperative day 4 (P4d); postoperative day 7 (P7d): postoperative month 3 (P3M); postoperative month 6 (P6M) 